\title{
RESEARCHES OF INTELLIGENT MEASURING DEVICES FOR CONSUMER PROTECTION
}

\author{
Genadijs Moskvins \\ Latvia University of Life Sciences and Technologies, Latvia \\ logicor@llu.lv
}

\begin{abstract}
The scientific work contains the researches on the automatic measuring system development problem including new types of devices and methods for rapid tests of quantity and quality of products by using microcomputers. Novel alternatives for improvement of intelligent measuring instruments are offered in the article. By using intelligent primary measuring transducers (PMT) on the basis of the statistic method an approach for control problem of consumer protection is shown. In the article the main results of the theoretic analysis and experimental researches of new possibilities to development of intelligent sensors and measuring instruments and the theoretical substantiations and practically useful conclusions are submitted. On the basis of the results of experimental researches a new method was developed for accuracy control and compact low- cost electronic devices for protection of consumers' rights.
\end{abstract}

Keywords: consumer protection, measuring devices.

\section{Introduction}

The report of the EU Food Fraud Network and the System for Administrative Assistance \& Food Fraud presents statistics for the year 2017 [1] on the cases exchanged in the AAC (Administrative Assistance and Cooperation System) and shows that, although there is no obligation to use the system, the competent authorities in the Member States have now taken the habit to use the system for requests regarding non-compliances and suspicions of food fraud to their counterparts in other Member States.

The EU has one of the highest food safety standards in the world - largely thanks to the solid set of the EU legislation in place, which ensures that food is safe for consumers. A key tool to ensure the flow of information enabling swift reaction when risks to public health are detected in the food chain is RASFF - the Rapid Alert System for Food and Feed (Fig.1).

\section{RASFFIACC FUTURE FLOW}

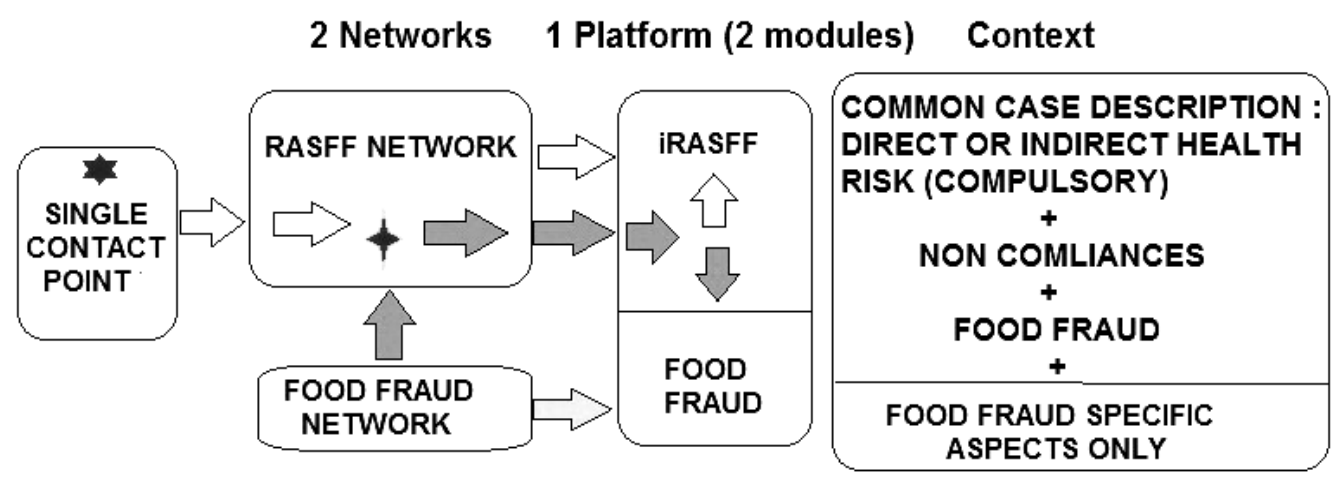

Fig. 1. RASFF/AAC future flow, adapted from [1]

RASFF continues to show its value to ensure food safety in the EU and beyond. Thanks to RASFF, many food safety risks can be averted before they could have been harmful to European consumers as a whole [2]. The measurement and evaluation of safety and quality is a complicated affair. In the most generic sense, quality refers to the combination of characteristics that are critical in establishing a product's consumer acceptability. But by means of the global RASFF system it is far not all food safety risks can be averted personally by each consumer enough operatively. Therefore, it is necessary to developed new rapid-testing methods and compact low-cost instruments for personal protection of each consumer.

Intelligent sensors can essentially perfect the whole control system due to the increase of preciseness and a rational processing of signals received from the sensory element. Fundamental research in the field of measuring systems allows creating abstract universal models which "improve" real processes and, in the result, the investigated situation becomes transparent, accessible to 
theoretical analysis, generalizations and for the defining of new knowledge. Intelligence of any type of sensors refers to any object in such a way as it is required by its nature [3-5].

Technological revolution in all the production spheres, especially in computing- and researchcomprising technology branches, determined the application of local systems with divided intellect in the functioning structures for the further development of intelligent sensors (IS). The elaboration of intelligent sensors becomes the main direction in development of intelligent measuring systems. That allows save recourses, also better protect interests and rights of consumers.

The high technology methods and devices contain programs of metrological certification and an estimation of accuracy, including the algorithms of error correction, also special interfaces for automatic data accumulation and accuracy analysis [3]. Figure 2 shows experimental smart devices for rapid testing of agricultural products.

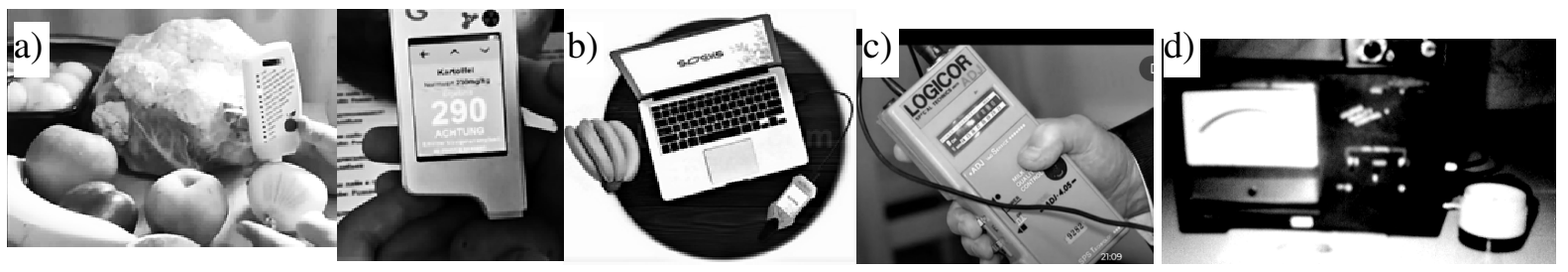

Fig. 2. Intelligent measuring devices for consumers' protection: $a-U K ; b-$ Germany; c - Canada; d, e - two variants of electronic tongue "Logicor-T", LLU

An e-tongue "Logicor-T" gives either a simple answer "good" or "bad", or a more sophisticated response, such as odour intensity or a molecule concentration. Sensors are immerged directly into the liquid or into others products. The aim of the scientific work is experimental researches on the possibilities of the new method and intelligent measuring instruments for consumer protection by simultaneous provision of mathematical, physical and metrological modelling of the optimal signal of the device at the measurement site in real conditions, which will allow to guarantee more accurate and reliable measurement results.

Besides the optimized applied programs, algorithms, "know-how" and processors, the full set of components for the system of intellectual measurements of quality and quantity of the consumed products is provided. At the same time, the consumer personally receives not only information, but also ready-to-use accurate and reliable knowledge about the quality or quantity of the consumed product. Such methodological approach in the elaboration of intelligent sensors very often in the measuring process does not provide a sufficiently complete idea about all the inner processes going on in the object under exploration, because the functional structure of the synthesized device is oriented only towards the realization of in advance programmed functions $[3 ; 6]$.

\section{Materials and Methods}

The research objects were primary measuring transducers (PMT), mathematical, physical and metrological models, programs and algorithms of intelligent (teaching, self-diagnostics) and metrological ensuring (evaluation, control and regulation of measuring results' precision and correction of errors), based on Ashby "s "black box approach" method and error correction algorithm [4].

The basic results were formed in a complete agreement of the existing notions with the level of knowledge about the investigation process with the trend being towards the convergence of development an intelligent technologies to estimate error factors and preciseness of sensors. Analytical research was focused on elaboration of mathematical models of digital intelligent measuring systems and statistic assessing of their condition.

Control of modelling results was carried out using methods described in detail in relevant USA and European patents $[3 ; 6]$. The experimental test of the elaborated methods of the preciseness control, metrological and analytical models, as well as the correction algorithm proved already accepted scientific theses and adequateness to physical models. Control of modeling results was carried out by the weight method. Figure 2 shows an algorithm of correction, as well as the microcomputer "Logicor-DS". 
a)

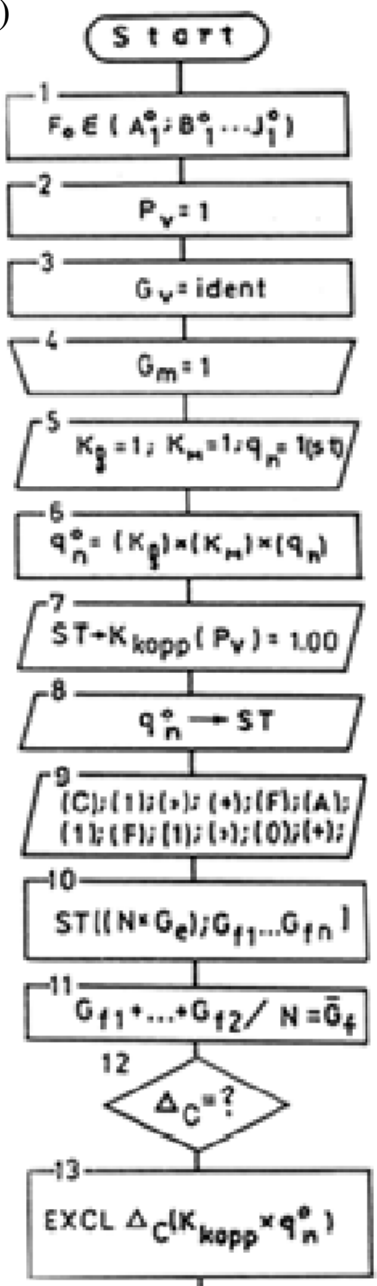

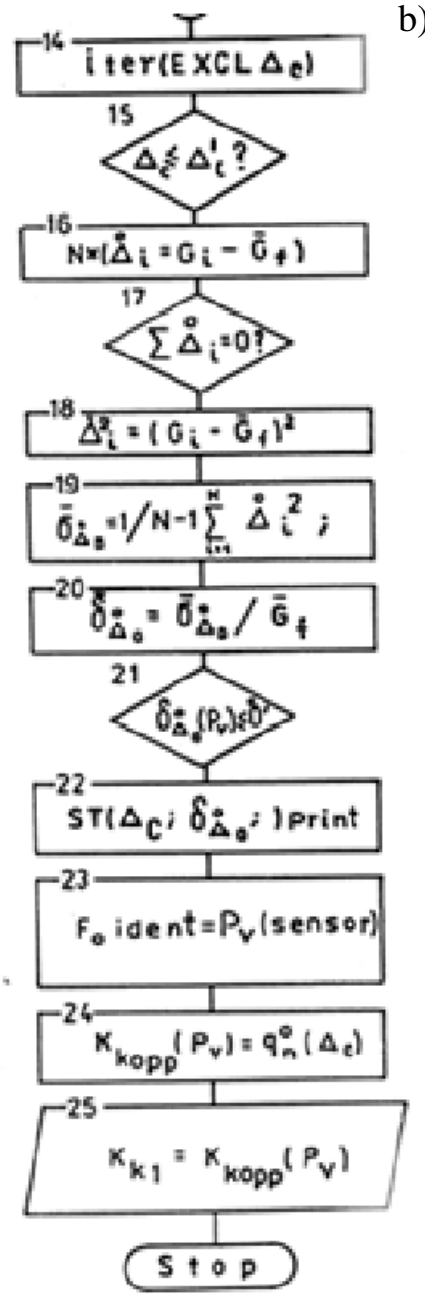

b)

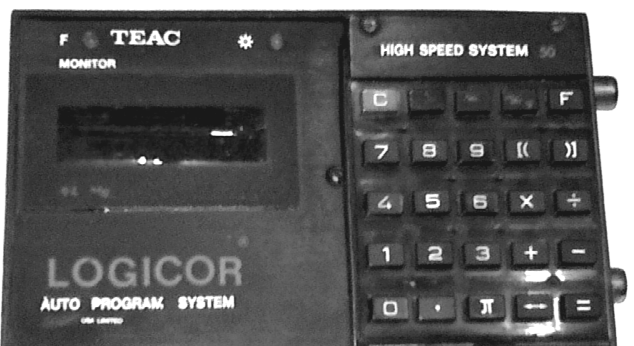

Fig. 3. Algorithm of self-calibration (a) and the microcomputer "Logicor-DS" (b)

(b)

\section{Results and Discussion}

The main task of the research was the metrological ensuring of the measuring channel "primary measuring transducer - microcomputer", substantiation and elaboration of structurally - functional ties of the intelligent measuring system. Simulation of an intelligent measuring system based on Ashby's "black box approach" allows analysis of the simulated system, taking into account behaviour of the research object as the response on the testing signals. For the assessment of the parameters of the target signal, a mathematical model with two keys of digital signals (basic and correction) was elaborated [3].

The correction signal of the measuring device in the form of a digital filter creates a digital impulse signal with modulation of the second type effects. To define the response of the measuring system, it is necessary to define $\mathrm{p}$ transformation of the entrance signal in the form of $G p(s)$, and then to calculate the Laplace transform for the target signal using the following formula [6]:

$$
X(s)=G p(s) Y(s) \text {. }
$$

Using special tables, it is possible to choose a standard-type signal of the working measuring device as a time function:

$$
\begin{gathered}
G(s)=1 / s, \\
Y(s)=1 /(s+a),
\end{gathered}
$$

where $\alpha$-factor of smoothing of a signal. 
It is also possible to define reaction of the system target signal as a result of $p$ transformation of the entrance signal:

$$
G_{p}(s)=P_{h, t}(1 / s),
$$

where $\mathrm{h}$ and $\mathrm{T}-$ indexes of time of an electronic key in the condition on and off.

From the last formula, according to the rules of $\mathrm{p}$ transformation, an equation is obtained:

$$
G_{p}(s)=\frac{1-e^{-h s}}{s\left(1-e^{-T s}\right)} .
$$

From basic formula (1) an equation of Laplace transform for the target signal is obtained:

$$
X(s)=G_{p}(s) Y(s)=\frac{1-e^{-h s}}{\left(1-e^{-T s}\right)} \frac{1}{s(s+a)} .
$$

From equation (6), the target signal can be defined as the sum of two shifted functions:

$$
L^{-1}\left\{\frac{1-e^{-h s}}{s(s+a)}\right\},
$$

where the interval of shift is equal to $0, t, 2 t$ and so on. It corresponds to the following ratio:

$$
\frac{1}{\left(1-e^{-s T}\right)}=1+e^{-s T}+e^{-2 s T}+\ldots+e^{-n s T} .
$$

The original of function $\mathrm{X}(\mathrm{s})$ is defined directly from the Laplace transform tables:

$$
X(t)=\left[1-d(-h)\left\{\frac{i}{\alpha}-\frac{1}{\alpha\left(e^{\alpha T}-1\right)}\left[e^{\alpha \sigma T}-e^{-\alpha T}\right]\right\} .\right.
$$

where $\mathrm{d}(-\mathrm{h})$ specifies the time delay.

Formula (9) allows calculating the correction signal of the intelligent measuring device at any time moment $\mathrm{T}$.

Considering that

$$
t=i T+\sigma T, i=0,1,2,3 \ldots, 0<\sigma<1
$$

at the numerical values $\alpha=1, T=0.5$, the final equation of the digital correction signal can be defined as follows:

$$
X(t)=[1-d(-h)]\left\{0,5 i \frac{1}{e^{0,5 \sigma}-1}\left[e^{0,5 \sigma}-e^{-T}\right]\right\} .
$$

Behaviour factor analysis of the object has to be considered at the basis of the experimental testing models. Further on reference functions, the influence factors and feedback algorithm in the form of programs of "self-learning" can be determined. Presence of specific measuring processes peculiarities caused the creation of such models of measuring systems, which at a higher rate would correspond to perspective tasks and aims both of artificial intelligence systems and measuring of physical magnitude with the account of the above mentioned fundamental problems of accuracy.

The peculiarities, conditions and specifics of food production require elaborate simple, safe, inexpensive and precise electronic conformity control devices. The elaboration of such devices control systems for the quality and quantity of resources is the decisive factor in operation of the conformity control for protection of consumers. The quality conformity assessment and respectively the risks in the rapid alert system in the chain of agricultural production can be easy detected by easy statistics methods. For assessing the accuracy of the intelligent measuring system using an algorithm of selfcalibration and the microcomputer automatically was calculated an optimal digital impulse of the sensor for calibrated values $5 ; 10 ; 100$ and $500 \mathrm{~kg}$ of the tested product.

In Fig. 4 the main results of researches by means of the microcomputer device "Logicor-DS" are reflected. They show that accuracy of measurements essentially depends on the value of a discrete impulse and volume or mass of the measured products. The main result of researches to solve the 
problem of optimization is the concordance of the artificial intelligence system building principles with the structure of the channel "PMT - microcomputer".
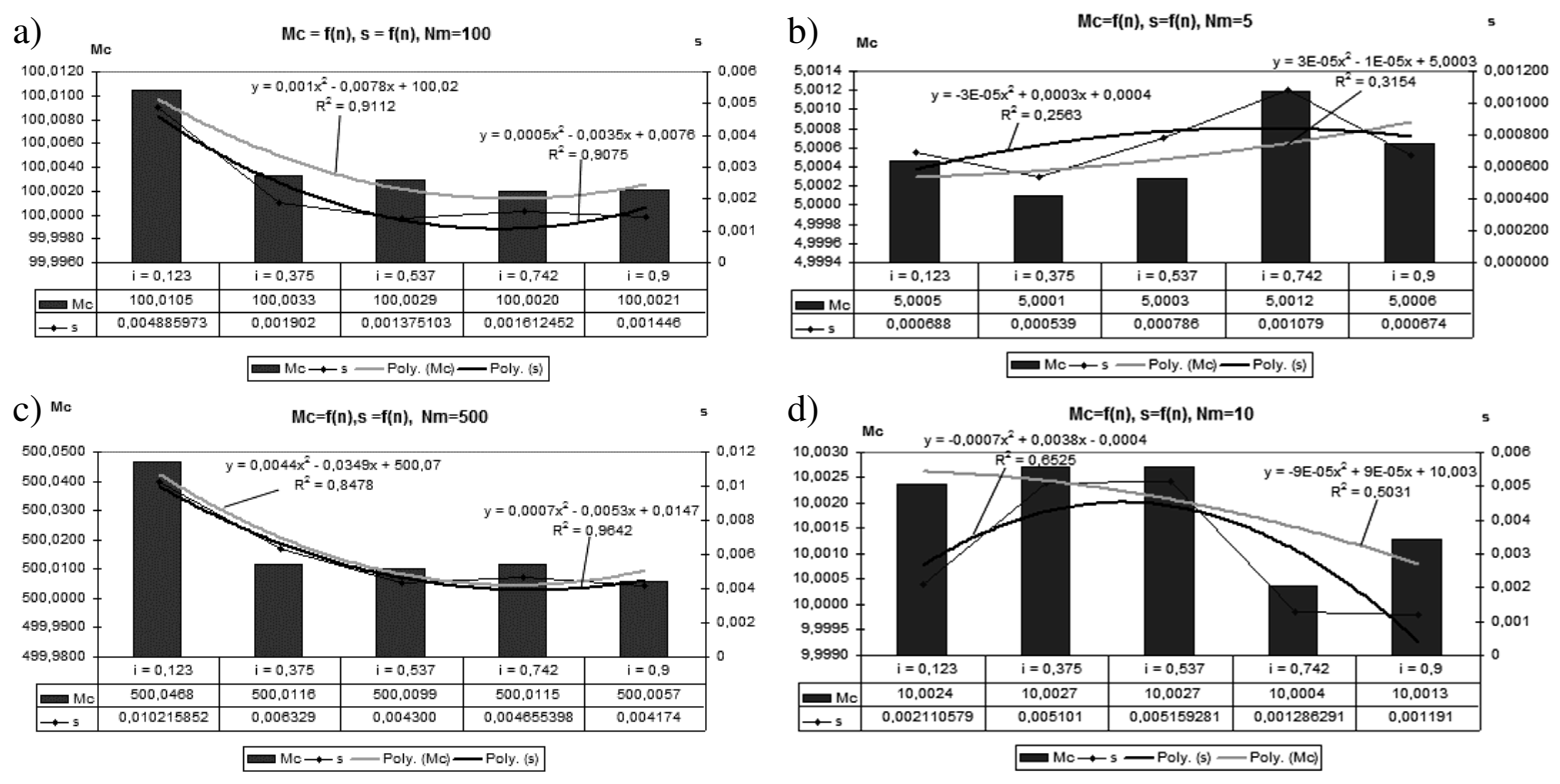

$$
\begin{aligned}
& \text { Average } \\
& M_{c}=\frac{\sum_{i=1}^{n} x_{i}}{n} \quad s=\sqrt{\frac{\sum_{i=1}^{n}\left(x_{i}-\bar{x}\right)^{2}}{n-1}}
\end{aligned}
$$

Fig.4. Simulation results of the optimal correction signal: $M c=f(n), S=f(n)$, $i=0,1,2,3 \ldots, 9 ; a-N m=100 \mathrm{~kg} ; b-N m=5 \mathrm{~kg} ; c-N m=500 \mathrm{~kg} ; d-N m=10 \mathrm{~kg}$

The offered new intelligent measuring processing principle will allow the consumers to receive more complete knowledge for monitoring of quantity and quality of products.

Compared to other intelligent measuring devices, the electronic devices "Logicor-DS" and "Logicor-T" are simpler, more accurate and more reliable. Besides, both devices contain a unit for analysis and correction of measurement results. Device "Logicor-T" contains also a unit for recognition of the product electronic code by means of a mobile application.

In Figure 5 examples of sausage product tests by means of e-tongue "Logicor-T" are reflected.

a)

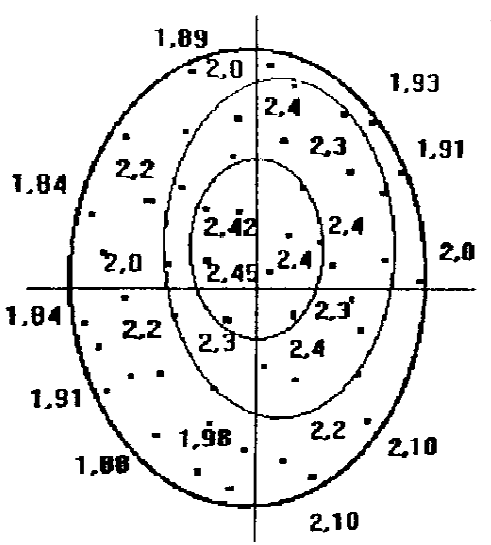

b)

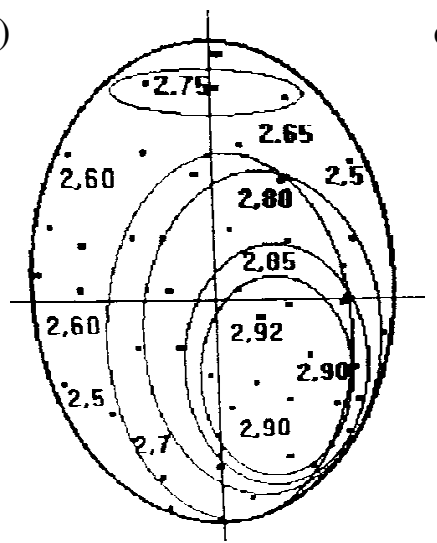

c)

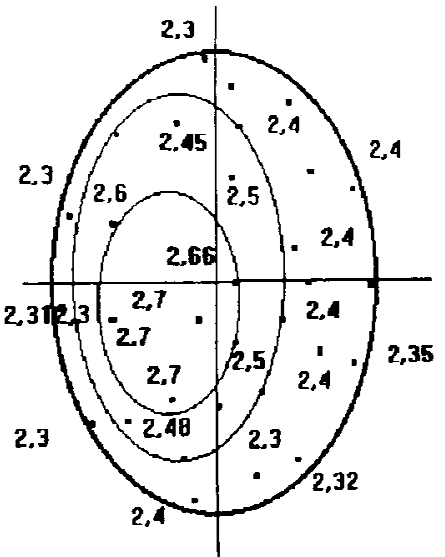

Fig. 5. Examples of sausage product tests by means of e-tongue "Logicor-T": a - sample N4/RGK, $\mathrm{NaCl}$, Norm index of quality $\mathrm{K}(\%)=2.4, \mathrm{~K} 1=1.84-2.20 ; \mathrm{K} 2=2.21-2.40 ; \mathrm{K} 3=2.41-2.45$; $\mathrm{b}$ - sample N-6a/RGK, K1 = 2.5-2.65; K2 = 2.66-2.70; K3 = 2.71-2.79; K4 = 2.8-2.84; K5 = 2.85-

$2.89 ; \mathrm{K} 6=2.90-2.92 ; \mathrm{c}-$ sample N-3a/RGK, $\mathrm{K} 1=2.3-2.4 ; \mathrm{K} 2=2.41-2.65 ; \mathrm{K} 3=2.66-2.70$ 


\section{Conclusions}

1. The new type of the intelligent measuring device "Logicor" in comparison with applied today devices allows to increase accuracy of measurements approximately in ten times.

2. The results of the research shown $\left(R^{2}=0.2-0.5\right.$ at values $N m=5-10 ; R^{2}=0.84-0.96$ at values $\mathrm{Nm}=100-500$ ) that the optimum value of the digital signal is not a constant value and has local deviations. The accuracy of the measurement depends on the mass of the measured value and requires a special block for statistics analysis and automatic correction of the measured signal. This scientific result is very important for the process of training of artificial neural networks (ANN), for example, for determining optimal weighting coefficients in back propagation algorithms (BPA) for elimination of local minimums of automatic control signals.

3. In the result of the analysis of modern traditional and untraditional-statistical methods the main reasons of errors are pointed out:

- different number of measurements;

- errors of mathematical, physical, statistical and metrological models inaccuracy;

4. On the basis of the experimental research such concordance for PMT is offered:

- intelligent measuring channel "PMT - microcomputer" can be elaborated as an expert system, opened for teaching and self-diagnostics with state of self-calibration possibility including programs and algorithms ensuring error correction;

- algorithms of smart measuring processors must include stages of indication, identification, interpretation, estimation and correction of measurement results.

\section{Acknowledgements}

Smart device "Logicor-DS" was awarded by Gold Medal of the International Salon of Inventions and Innovations Technologies Archimedes (Salon Archimedes, Moscow).

\section{References}

[1] RASFF - The Rapid Alert System for Food and Feed - 2018 annual report. Luxembourg: Publications Office of the European Union, ISBN 978-92-76-02635-8; ISSN 1830-7302, 2019;

[2] Conflicting Notifications in the EU's Rapid Alert System for Food and Feed (RASFF): "Destabilization" in Food Risk Communication? Published online by Cambridge University Press: 20 January 2017. DOI: 10.1017/S1867299X00000957

[3] Moskvins G. Intelligent chemical and physical conformity assessment tools. ISBN 978998488533/Catalogue of the 7th International Invention and Innovation Exhibition MINOX 2018/CONNECT Latvia. Riga, 2018, pp.14-15;

[4] Sandhusen R.L. Black-box theory used to understand Consumer behaviour Marketing.

[5] Moskvin G., Spakovica E. Intelligent Technology for the Conformity Assessment of Agricultural Products. Advances in Computer, Information, and Systems Sciences, and Engineering. Hardcover ISBN: 1-4020-5260-X, Springer Berlin-Heidelberg -New York, 2006, pp. 109-114;

[6] Moskvins G., Spakovica E., Moskvins A., Sahtarina A. Mathematical Modelling of the Digital Measuring Signal of Intelligent Flowmeters, Proceedings of the Latvia University of Agriculture, 29(324), 2013, pp.41-47. DOI: 10.2478/v10236-012-0022-1 